Bundesgesundheitsbl -
Originalien und Übersichtarbeiten

I. Klare · C. Konstabel · D. Badstübner · G. Werner · W. Witte

Robert Koch-Institut, Berlin

\title{
Glycopeptid-resistente Enterokokken in deutschen Krankenhäusern 1998
}

\section{Zusammenfassung}

Es wurden 92 Stämme Glycopeptid-resistenter Enterokokken (GRE) von 80 Patienten aus 31 Krankenhäusern in elf Bundesländern analysiert. GRE wurden vorrangig in den intensivmedizinischen Bereichen (allgemeine, chirurgische, internistische, pädiatrische, onkologische ITS-Stationen), aber auch in $\mathrm{Ne}$ phrologie/Dialyse- oder Neurologie/Orthopädie-Abteilungen isoliert. Dabei war die Dominanz von VanA-E. faecium-Stämmen zu beobachten $(n=81 ; 88,0 \%)$, weiterhin wurden Stämme von E. faecalis (VanA; $n=4$; $4,4 \%$ ), E. faecium ( $\operatorname{Van} B ; n=1 ; 1,1 \%$ ) sowie E. gallinarum (VanC1; $n=6 ; 6,5 \%$ ) gefunden. Die 81 VanA-Stämme von E. faecium zeigten folgende Resistenzquoten gegen weitere Antibiotika: Erythromycin, Ciprofloxacin (jeweils 93,8\%), Ampicillin, Oxytetracyclin (je 88,9\%), Rifampicin (79,0\%), Trimethoprim/Sulfamerazin $(61,7 \%)$, Chloramphenicol (18,9\%), Fusidinsäure (12,3\%), Quinupristin/Dalfopristin (7,4\%); Gentamicin (46,9\%) und Streptomycin (37,0\%), auch kombiniert mit Ampicillinresistenz (9,9-35,8\%). Bei einigen dieser VanA-Stämme war die Resistenz gegen Teicoplanin in-vitro nicht vollständig exprimiert. Gleiche Makrorestriktionsmuster von E.faecium-Ausbruchsstämmen (VanATyp) aus Krankenhäusern in verschiedenen Bundesländern deuteten auf eine intra- und interhospitale Verbreitung eines definierten GRE-Stammes. Analysen der Plasmid- und Plasmidrestriktionsmuster dieser Isolate zeigten jedoch deutliche Unterschiede. Dies bedeutet, daß E.faecium-Isolate mit gleichem Makrorestriktionsmuster nicht unbedingt identisch sein müssen.

\section{Schlüsselwörter \\ Enterokokken · Glycopeptidresistenz · Ausbrüche · Genotypisierung}

Enterokokken gehören zu den wichtigsten Erregern nosokomialer Infektionen und nehmen heute mit 12\% des Auftretens nach Escherichia coli und Staphylococcus aureus Rang 3 der durch Bakterien hervorgerufenen Krankenhausinfektionen ein. Dabei ist im allgemeinen ein Dominieren von E. faecalis (8o bis $90 \%$ der Isolierungen) gegenüber E. faecium (10 bis $20 \%$ ) zu beobachten. Allerdings kann insbesondere in intensivmedizinischen Bereichen mit hohem Antibiotikaeinsatz dieses Verhältnis zugunsten von E. faeci$u m$ verschoben sein, da diese Spezies über ein breiteres Spektrum an natürlichen und erworbenen Antibiotikaresistenzen verfügt. Seit etwa zehn Jahren erwecken Enterokokken mit Glycopeptidresistenz weltweit ein besonderes Interesse. Erstmals beschrieben wurden diese Bakterien 1988 zeitgleich in Frankreich und Großbritannien $[1,2]$. Sie sind heute in den Kliniken (insbesondere auf inten- sivmedizinischen Stationen) in Abhängigkeit vom jeweiligen Antibiotika-Selektionsdruck mit unterschiedlicher Häufigkeit zu finden. So wurde beispielsweise in den Vereinigten Staaten im Zeitraum 1989 bis 1997 ein Anstieg des Anteils der GRE innerhalb der Enterokokken von 0,3\% (1989) auf 7,9\% (1993) und schließlich auf 15,3\% (1997) in Normalstationen beobachtet; im intensivmedizinischen Bereich war dieser Anstieg im gleichen Zeitabschnitt noch rapider: 0,4\% (1989), 13,6\% (1993), 23,2\% (1997); auf einigen Stationen bis $43 \%[3,4]$.

\section{„Abhängig vom Antibiotika- Selektionsdruck sind Glycopeptid- resistente Enterokokken heute in Kliniken weltweit in unter- schiedlicher Häufigkeit zu finden."}

In Europa ist die Situation hinsichtlich des Auftretens von GRE noch vergleichsweise günstig, so liegt der Anteil Glycopeptid-resistenter Enterokokken meist unter $5 \%$, oftmals weniger als $2 \%[5,6]$. Dabei ist jedoch zu berücksichtigen, daß einerseits die Mehrzahl der Enterokokkeninfektionen durch E. faecalis verur-

\section{Dr. Ingo Klare}

Robert Koch-Institut, Postfach 6502 80,

D-13303 Berlin 
Bundesgesundheitsbl -

Gesundheitsforsch - Gesundheitsschutz

1999 · 42: 847-853 @ Springer-Verlag 1999

I. Klare · C. Konstabel · D. Badstübner · G.Werner $\cdot$ W.Witte

\section{Glycopeptide-resistant enterococci in German hospitals in 1998}

\section{Summary}

Ninety-two strains of glycopeptide-resistant enterococci (GRE) from 80 patients of 31 hospitals in 11 federal states were analyzed. They were primarily isolated in intensive care units (general, surgical, internistic, pediatric, oncological ones), but also in nephrolo$\mathrm{gic} /$ dialysis or neurologic/orthopaedic wards. A dominance of VanA type E. faecium strains was observed ( $n=81 ; 88.0 \%)$. In addition, strains of $E$. faecalis (VanA; $n=4 ; 4.4 \%)$, E. faecium (VanB; $n=1 ; 1.1 \%$ ), and E. gallinarum (VanC1, $n=6 ; 6.5 \%$ ) were found. The 81 VanA strains of $E$. faecium possessed the following resistances to other antibiotics: erythromycin, ciprofloxacin (both 93.8\%), ampicillin, oxytetracycline (both $88.9 \%$ ), rifampicin (79.0\%), trimethoprim/sulfameracin (61.7\%), chloramphenicol (18.9\%), fusidic acid (12.3\%), quinupristin/dalfopristin (7.4\%); gentamicin (46.9\%) and streptomycin (37.0\%), also together with ampicillin resistance (9.9-35.8\%). In some of these VanA type strains resistance to teicoplanin cannot be completely expressed in-vitro. Macrorestriction analysis of $E$. faecium outbreak isolates (VanA type) from hospitals in different federal states indicated an intra- and interhospital spread of a defined strain. However, heterogeneous plasmid and plasmid restriction patterns showed that these strains were not completely identical.

\section{Key words}

Enterococci - Glycopeptide resistance Outbreaks · Genotyping

\section{Originalien und Übersichtarbeiten}

sacht wird (siehe oben), andererseits E. faecium das Hauptreservoir für die übertragbare(n) Glycopeptidresisten$\mathrm{z}(\mathrm{en})$ ist. Daneben existiert auch die Möglichkeit des Einschleppens von GRE in die Krankenhäuser von außerhalb. Dies kann entweder über kontaminierte Fleischprodukte oder über Patienten geschehen, die bereits GRE in ihrem Darm tragen (Rolle der Nahrungskette im Zusammmenhang mit der Avoparcinanwendung in der kommerziellen Tiermast [7-13]).

\section{Material und Methoden}

\section{Bakterienstämme}

Von den im Jahr 1998 an das Robert Koch-Institut (Bereich Wernigerode) eingesandten Enterokokkenisolaten ( $n=369)$ konnten insgesamt 92 GREStämme in die Auswertungen einbezogen werden. Bei den übrigen Stämmen handelt es sich zum Großteil um Glycopeptid-sensible Isolate, die zu derzeit noch laufenden Untersuchungen bezüglich Kreuzinfektionen bei Krankenhauspatienten gehören. Die Auswahl der 92 GRE-Stämme erfolgte entsprechend des Vorliegens von jeweils unterschiedlichen Makrorestriktionsmustern (Vermeidung von Copy-Isolaten). Diese 92 Stämme wurden bei 80 Patienten aus 31 Krankenhäusern in elf Bundesländern (Baden-Württemberg, Bayern, Berlin, Brandenburg, Hamburg, Mecklenburg-Vorpommern, Niedersachsen, NordrheinWestfalen, Sachsen, Sachsen-Anhalt, Schleswig-Holstein) isoliert (Tabelle 1).
Die Identifizierung der Bakterien erfolgte entsprechend der Vorgehensweise von Devriese et al. [14]. Die Resistenzbestimmung wurde durch Ermittlung minimaler Hemmkonzentrationen ( $\mathrm{MHK}$ ) in Isosensitest-Bouillon mittels Mikrobouillonverdünnungstest durchgeführt [15]. Es wurden folgende Antibiotika geprüft und entsprechend der nachfolgenden MHK-Grenzen $(\mu \mathrm{g} / \mathrm{ml})$ für sensibel (s) bzw. resistent (r) ausgewertet: Benzylpenicillin ( $s \leq 0,125 ; r \geq 2)$; Ampicillin und Sulbactam-geschütztes Ampicillin $(s \leq 2 ; r \geq 16$; Sulbactam in konstanter Konzentration von $8 \mu \mathrm{g} / \mathrm{ml}$ getestet); Gentamicin ( $s \leq 1$; $r \geq 8$; Hochresisten $z \geq 1024$ ); Streptomycin ( $s \leq 8 ; r \geq 32$; Hochresistenz $\geq 2048$ ); Erythromycin, Clindamycin, Oxytetracyclin (jeweils $s \leq 1 ; r \geq 8$ ); Chloramphenicol $(s \leq 8 ; r \geq 16)$; Vancomycin, Teicoplanin (jeweils $s \leq 4 ; r \geq 16$ ), Ciprofloxacin $(\mathrm{s} \leq 0,25 ; \quad \mathrm{r} \geq 2)$; Trimethoprim/Sulfamerazin ( $s \leq 4 ; r \geq 32)$; Rifampi$\operatorname{cin}(s \leq 0,5 ; r \geq 1)$; Fusidinsäure $(s \leq 2 ; r \geq 4)$; Quinupristin/Dalfopristin $(s \leq 1 ; r \geq 4)$.

\section{Nachweis der van-Glycopeptidresis- tenzgene und der sat-Streptogramin- resistenzgene von Enterokokken mittels PCR}

Die Gene vanA bzw. vanB wurden mittels PCR entsprechend einer früheren Vorschrift [16], die vanC1- und vanC2Gene nach der Methode von Klare et al. [17] detektiert. Dabei wurde anstelle des bei [17] angegebenen reverse-Primers für vanC2 der folgende Primer verwendet: 5' CGA GCA AGA CCT TAA AG 3'. Die Gene für die bei Enterokokken

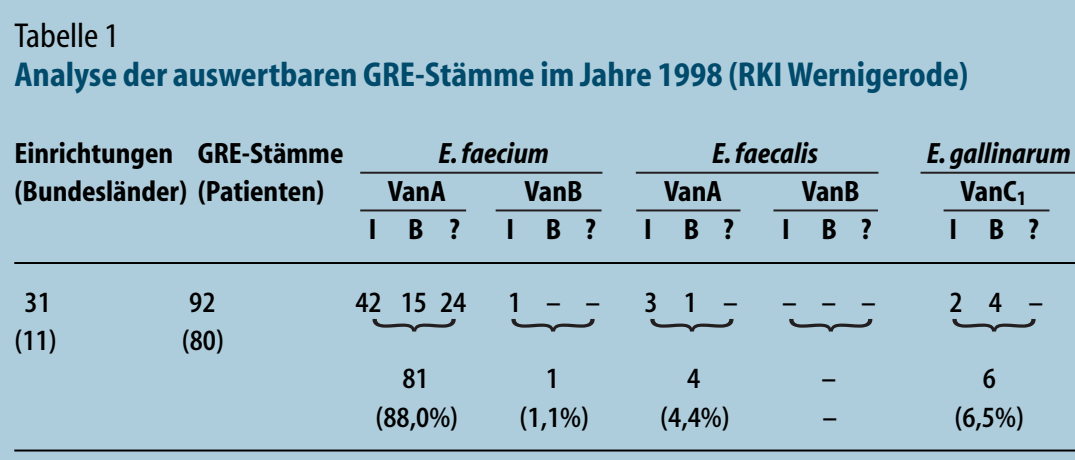

I:Infektionen, B: Besiedlungen, ?: unklare Angaben 
beschriebenen Streptogramin-Acetyltransferasen sat A bzw. sat $G$ wurden durch PCR entsprechend $[18,19]$ nachgewiesen.

\section{Genotypisierung der Enterokokkenisolate}

Die genotypische Charakterisierung (DNA-Fingerprinting) der Enterokokkenstämme wurde mittels Makrorestriktionsanalyse (SmaI-Fragmentmuster; in speziellen Fragestellungen auch zusätzlich ApaI-Fragmentmuster) entsprechend der bereits früher beschriebenen Methodik von Klare et al. [10, 21] und Claus et al. [20] durchgeführt. Bakterielle Isolate mit $\leq 3$ DNA-Banden Unterschied im Makrorestriktionsmuster sind entsprechend Tenover et al. [22] als identisch bzw. sehr nahe verwandt zu bewerten.

\section{Isolierung und Charakterisierung der Plasmide}

Die Plasmidisolierung erfolgte nach Woodford et al. [23] und modifiziert nach Werner et al. [24]. Die nichtgespaltenen und EcoRI-gespaltenen Plasmide wurden in o,8\%igen Agarose-Gelen elektrophoretisch aufgetrennt und anschließend auf eine positiv geladene $\mathrm{Ny}$ lonmembran (Boehringer, Mannheim) geblottet. Eine Digoxigenin-markierte Sonde des vanA-Gens von E. faecium BM4147 (Referenzstamm für vanA) wurde mittels PCR entsprechend Werner et al. [25] hergestellt.

\section{Ergebnisse und Diskussion}

\section{Spezies, Glycopeptid-Resistenztypen und klinische Herkunft der Enterokokken}

Von den ausgewerteten 92 GRE-Stämmen war die überwiegende Mehrheit der Isolate der Spezies E. faecium $(n=82$; $89,1 \%)$ zuzuordnen, E. faecalis war mit vier Stämmen (4,4\%), E. gallinarum mit sechs Stämmen $(6,5 \%)$ vertreten. In den meisten Fällen bestätigte die Speziesdiagnostik in unserem Labor die Ergebnisse der Einsender. Schwierigkeiten gab es bei wenigen E. faecium-Isolaten, die uns
Tabelle 2

Aufschlüsselung der 42 VanA-Typ E. faecium-Stämme aus

Infektionen auf die klinischen Bereiche (RKI Wernigerode, 1998)

\begin{tabular}{l} 
Bereich \\
\hline Intensivstation (allg.) \\
Internistische Intensivstation \\
(inkl.Transplantationseinheiten) \\
Chirurgische Intensivstation \\
Onkologische Intensivstation \\
Pädiatrie (Intensivstation) \\
Nephrologie/Dialyse (einschl. Kinder-Dialyse) \\
Gynäkologie \\
Neurologie/Orthopädie \\
keine Angaben \\
Sumbulant (Harnwegsinfektion) \\
Summe
\end{tabular}

$n$ Stämme

6

6
7

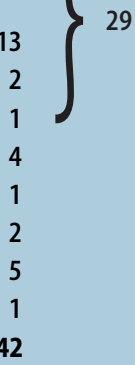

fälschlicherweise als „Vancomycin/Teicoplanin-hochresistente E. gallinarumbzw. E. casseliflavus-Stämmen" eingesandt wurden. Ursache dieser Fehlbestimmungen sind offensichtlich das Nichtvorhandensein der wichtigen Tests auf Beweglichkeit und gelbes Pigment in einigen kommerziellen Identifizierungskits. Von sehr seltenen Ausnahmen einmal abgesehen, sind die Spezies der sog. E. gallinarum-Gruppe (E.gallinarum, E. casseliflavus, E. flavescens) beweglich, und die zwei letztgenannten Arten verfügen zusätzlich über ein gelbes Pigment. Dieses gelbe Pigment ist zwar auch bei E. mundtii zu finden, jedoch ist diese Spezies unbeweglich. E. faecium ist unbeweglich und besitzt kein Pigment. Ähnliche Schwierigkeiten bei der korrekten Speziesbestimmung traten auch in einer von unserer Arbeitsgruppe geleiteten Ringstudie zur Identifizie-rung und Glycopeptidresistenzbestimmung von Enterokokken durch klinisch-mikrobiologische Laboratorien in Deutschland und Österreich auf [26].

Bezogen auf die Glycopeptidresistenztypen dominierte der VanA-Typ von E. faecium (81 von 92 EnterokokkenStämmen; 88,0\%), nur ein Stamm dieser Spezies $(1,1 \%)$ zeichnete sich durch den VanB-Typ aus. Die genannten vier E. faecalis-Stämme gehörten zum VanA-Typ, und die sechs E. gallinarum-Stämme waren der natürlichen Glycopeptidresistenz des VanC1-Typs zuzuordnen (Tabelle 1). In dieser Tabelle mit enthalten ist die Einteilung der Isolate als Infektionserreger („I“), Besiedlungskeim („B“) bzw. Isolate mit unklarer Klassifizierung („?“). Da wir in erster Linie an GRE aus Infektionen interessiert sind, liegt in dieser Tabelle ein etwa gleiches Verhältnis der Stämme aus Infektionen $(n=48)$ gegenüber jenen als Besiedler+GRE mit unklarer Einteilung $(n=44)$ vor. In der täglichen klinisch-mikrobiologischen Praxis ist jedoch eher ein Überwiegen von GRE-Isolaten als Besiedlungskeime festzustellen. Die von uns ebenfalls untersuchten Stämme aus Besiedlungen gelangten bezüglich der Abklärung möglicher Infektionsketten zur Einsendung.

\section{"Die für Infektionen verantwort- lichen Glycopeptid-resistenten Enterokokkenisolate finden sich vor allem bei Patienten aus intensivmedizinischen Bereichen und der Nephrologie/Dialyse."}

Bei den in Tabelle 1 aufgeführten, durch E. faecium-Stämme des VanA-Typs bedingten Infektionen $(n=42)$ waren Wundinfektionen einschließlich Abszesse $(n=20)$, Harnwegsinfektionen $(n=11)$, Septikämien $(n=8)$ und Peritonitiden $(n=3)$ von den Einsendern angegeben worden. Tabelle 2 zeigt die Unterteilung 


\begin{tabular}{|c|c|c|}
\hline \multirow[t]{2}{*}{ Antibiotikum bzw. Antibiotika-Gruppe } & \multicolumn{2}{|c|}{ Resistenzraten } \\
\hline & $n$ & (\%) \\
\hline VAN & 80 & $(98,8)$ \\
\hline TPL & 69 & $(85,2)$ \\
\hline AMP & 72 & $(88,9)$ \\
\hline GEN & 38 & $(46,9)$ \\
\hline STR & 30 & $(37,0)$ \\
\hline AMP+GEN & 29 & $(35,8)$ \\
\hline $\mathrm{AMP}+\mathrm{STR}$ & 17 & $(21,0)$ \\
\hline $\mathrm{AMP}+\mathrm{GEN}+\mathrm{STR}$ & 9 & $(9,9)$ \\
\hline GEN+STR & - & - \\
\hline ERY & 76 & $(93,8)$ \\
\hline RAM & 64 & $(79,0)$ \\
\hline Q/D & 6 & $(7,4)$ \\
\hline OTE & 72 & $(88,9)$ \\
\hline CMP & 15 & $(18,9)$ \\
\hline CIP & 76 & $(93,8)$ \\
\hline SXT & 50 & $(61,7)$ \\
\hline FUS & 10 & $(12,3)$ \\
\hline
\end{tabular}

dieser 42 E. faecium-Stämme hinsichtlich ihrer klinischen Herkunft. Wie daraus zu erkennen ist, waren es vor allem Patienten aus den intensivmedizinischen Bereichen und der Nephrologie/Dialyse, bei denen diese GRE-Stämme gefunden wurden. Dies entspricht auch den allgemeinen Erfahrungen: mit einem besonderen Risiko für GRE-Infektionen sind (immunsupprimierte) Patienten in onkologischen, hämatologischen, nephrologischen, chirurgischen und Transplantationseinheiten (Intensivstationen) behaftet $[27,28]$. Ein E. faecium-Stamm (VanA) wurde im ambulanten Bereich bei einem Patienten mit Harnwegsinfektion isoliert.

\section{Antibiotikaresistenzen}

In Tabelle 3 ist die In-vitro-Resistenzsituation der insgesamt 81 VanA-Typ $E$. faecium-Isolate dargestellt. 88,9\% der untersuchten VanA-Isolate von E. faecium erwiesen sich als Ampicillin-resi- stent. Damit würde dann auch die ansonsten bewährte Kombinationstherapie aus Ampicillin und einem Aminoglycosid (Gentamicin, Streptomycin) versagen. Schon bei Vorliegen von Resistenz gegen einen dieser Kombinationspartner kommt es zum Scheitern des Wirkungssynergismus beider Antibiotika. Daneben verfügte ein beträchtlicher Anteil der E. faecium-Stämme über Hochresistenz gegen die zuvor genannten Aminoglycoside (Gentamicin: 46,9\%; Streptomycin: 37,0\%). Außerdem waren diese Hochresistenzen gekoppelt mit Ampicillin-Resistenz bei 9,9 bis $35,8 \%$ der Stämme (Tabelle 3). Jeweils 93,8\% der VanA-E. faecium-Stämme waren resistent gegen Erythromycin bzw. Ciprofloxacin, sehr hohe Resistenzquoten lagen auch gegen Rifampicin $(79,0 \%)$, Oxytetracyclin $(88,9 \%)$ und Trimethoprim/Sulfamerazin $\quad(61,7 \%)$ vor. Bei der Endokarditis kommt es mit der letztgenannten Antibiotika-Kombination jedoch zum Therapie-Versagen
[29]. Nur 15 von 81 Stämmen $(18,9 \%)$ zeigten Resistenz gegen Chloramphenicol (Tabelle 3), allerdings wirkt dieses Antibiotikum nur bakteriostatisch. Dennoch gibt es Berichte über teilweise erfolgreiche Behandlung von Infektionen durch vanA-positive E. faecium-Stämme mit diesem Antibiotikum [29,30]. In anderen Fällen wurde von einem Versagen der Chloramphenicol-Therapie bei Bakteriämie-Patienten berichtet [30-32].

\section{Expression der Glycopeptidresistenz}

69 der 81 VanA-E. faecium-Stämme $(85,2 \%)$ zeigten mit $\geq 16 \mu \mathrm{g} / \mathrm{ml}$ MHKWerte für Teicoplanin im resistenten Bereich (Tabelle 3). Die restlichen zwölf Stämme besaßen intermediäre Resistenzen (MHK: $8 \mu \mathrm{g} / \mathrm{ml}, n=9$ ), aber drei Stämme waren mit MHK-Werten von $4 \mu \mathrm{g} / \mathrm{ml}$ Teicoplanin-“sensibel“. Diese MHK-Werte der Enterokokken mit intermediärer bzw. an der Grenze zwischen sensibler und intermediärer Empfindlichkeitskategorie sprechen für eine unterschiedlich starke Expression der Glycopeptidresistenz. Enterokokken ohne vanA-Resistenzgencluster zeigen TeicoplaninMHK-Werte von 0,125-1 $\mu \mathrm{g} / \mathrm{ml}$. Bei den drei Stämmen mit MHK-Werten von 4 $\mu \mathrm{g} / \mathrm{ml}$ („sensibel“) war die TeicoplaninResistenz nur bei einem Teil der Population (ca. 10 ${ }^{-5}$ ) exprimiert (bisher unveröffentlichte Daten). Ob dies Bedeutung für die Therapie hätte, läßt sich nur schwer beurteilen. Kommen an Infektionsorten, die für Glycopeptide schwer erreichbar sind, hohe Keimkonzentration des betreffenden Enterokokkenstammes (und somit auch genügend resistente Zellen) vor, könnte dies zum Versagen der Glycopeptidtherapie führen.

\section{Resistenz gegen Quinupristin/ Dalfopristin}

Die Antibiotikakombination Quinupristin/Dalfopristin (Q/D) wirkt gut auf $E$. faecium und verwandte Arten (jeweils einschließlich GRE-Stämme) und erweitert damit das Therapiespektrum gegen multiresistente Isolate; E. faecalis besitzt eine natürliche Resistenz [33-35]. Bereits vor der klinischen $\mathrm{Zu}-$ lassung von Q/D waren $7,4 \%$ der hier 
untersuchten $E$. faecium-Stämme des VanA-Typs resistent gegen diese Streptogramin-Kombination. Die molekulare Ursache liegt in einer Inaktivierung der A-Komponente (bei Q/D: Dalfopristin) durch Acetylierung, was zu einem Verlust der synergistischen Wirkung beider Komponenten führt. Die erworbenen Acetyltranferase-Gene satA bzw. sat $G$ wurden bei den in Tabelle 3 aufgeführten Q/D-resistenten Stämmen mittels PCR nachgewiesen $[18,19]$. Q/D-resistente E. faecium-Isolate konnten auch aus Gülleproben kommerzieller Tiermastbetriebe isoliert werden, in denen die Streptogramin-Kombination Virginiamycin (aus den Komponenten S und $M$ bestehend) als „Leistungsförderer“ eingesetzt wurde. Q/D und Viginiamycin S/M sind strukturell sehr eng verwandt und selektieren Kreuzresistenz $[18,19,36]$. Des weiteren wurden Q/Dresistente E. faecium-Isolate in Fleischproben von Mastgeflügel, in Stuhlproben nicht hospitalisierter Personen sowie in städtischen Abwässern gefunden ([19] und unveröffentlichte Daten). Dies ist ein Hinweis darauf, daß außerhalb der Krankenhäuser bereits vor einer klinischen Zulassung von Q/D ein großes Reservoir an Streptogramin-resistenten E. faecium existiert.

\section{„Die Resistenz gegen das als Reservemittel Quinupristin/ Dalfopristin zur Behandlung von Enterokokkeninfektionen wird durch den Einsatz verwandter Substanzen als Leistungsförderer in der Tiermast begünstigt."}

Ein Zusammenhang zwischen dem nutritiven Antibiotikaeinsatz in der kommerziellen Tiermast und der Selektion Antibiotika-resistenter Krankheitserreger sowie der anschließenden Verbreitung dieser Isolate (oder ihrer Resistenzgene) über die Nahrungskette auf den Menschen wurde bereits am Beispiel der Streptothricinresistenz (Nourseothricin) bei gramnegativen Bakterien $[37,38]$ bzw. der Glycopeptidresistenz (Avoparcin) von Enterokokken gezeigt [7-13].
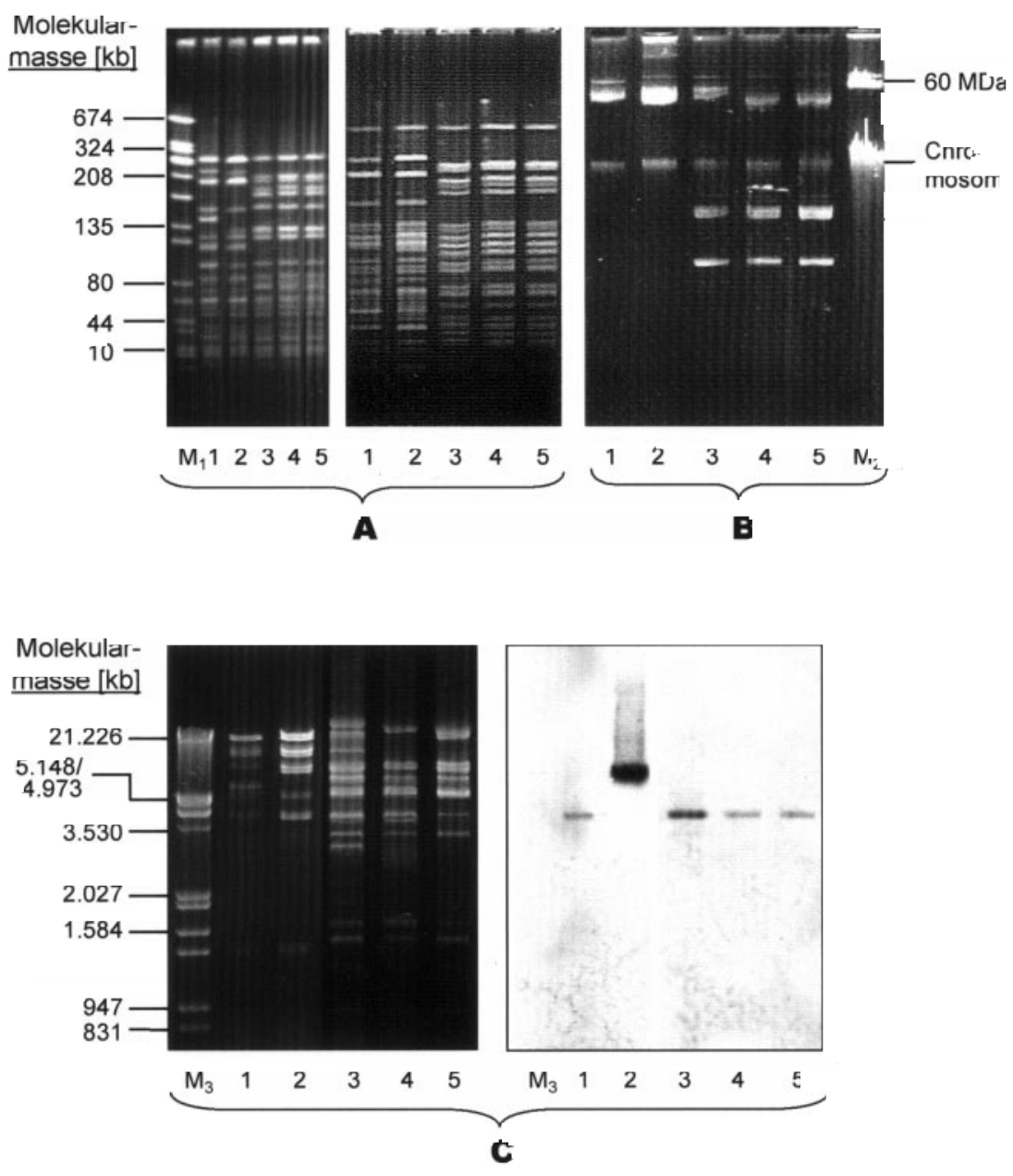

Abb. $1 \Delta$ Nachweis unterschiedlicher vanA-Plasmide von E. faecium-Isolaten mit gleichen SmalMakrorestriktionsmustern (VanA-Typ-GRE aus Ausbrüchen von Infektionen in verschiedenen deutschen Krankenhäusern)

A Makroestriktionsmuster nach Spaltung der genomischen DNA mittels Smal (links) bzw. Apal (rechts) B Plasmidmuster

C EcoRI-Restriktionsmuster der Plasmide (links) und anschließende Southern-blot-Hybridisierung mittels einer Digoxigenin-markierten Sonde des vanA-Gens (rechts) aus E.faecium BM 4147 (Referenzstamm)

\begin{tabular}{lll} 
Ifd. Nr. & Stamm & Herkunft: Krankenhäuser in \\
\hline 1 & UW 400 & Bayer \\
2 & UW 805 & Hessen \\
3 & UW 901 & Niedersachsen \\
4 & UW 931 & Nordrhein-Westfalen \\
5 & UW 1505 & Molekularmassenstandard (S. aureus NCTC 8325) \\
\hline$M_{1}$ & Molekularmassenstandard (E. coli R222) \\
$M_{2}$ & Molekularmassenstandard III (Boehringer Mannheim) \\
$M_{3}$ &
\end{tabular}




\section{Ausbrüche von GRE-Infektionen}

Neben dem sporadischen Auftreten von GRE-Stämmen ist das vermehrte Vorkommen von Isolaten von besonderem Interesse, die in einem epidemiologischen Zusammenhang stehen (Ausbrüche von GRE-Infektionen und -Besiedlungen). Im Jahr 1998 wurden uns E. faecium-Isolate (VanA-Typ) aus einem Ausbruch in einem Krankenhaus in Nordrhein-Westfalen zugesandt. Diese GRE-Isolate stammten von Patienten aus dem Bereich der Inneren Medizin bzw. Chirurgie [39]. Im gleichen Jahr sind auch im Berliner Raum bei Patienten verschiedener Stationen mehrerer Krankenhäuser Besiedlungen bzw. Infektionen durch einen definierten VanATyp E. faecium-Stamm beobachtet worden. Ausgangspunkt war hier offenbar ein Transplantationszentrum, von dem aus die Patienten nach ihrer Rückverlegung in die Heimatkrankenhäuser den entsprechenden GRE-Stamm transferierten (unveröffentlichte Daten).

Bei zurückliegenden Ausbrüchen von Infektionen und Besiedlungen durch E. faecium-Isolate des VanA-Typs in deutschen Krankenhäusern (zwei Kliniken in Bayern, je eine in Hessen und Niedersachsen) wurden GRE zuerst in Nephrologie/Dialyse-Stationen isoliert. Nachfolgend kam es zum Nachweis dieser multiresistenten Erreger bei Patienten in den oben genannten intensivmedizinischen und anderen Klinikbereichen [40-42]. Die mittels Makrorestriktionsanalyse durchgeführte Genotypisierung der VanA-E. faecium-Stämme (jeweils ein repräsentatives Isolat aus allen oben genannten Ausbrüchen [39-42]) zeigte, abgesehen von den Stämmen aus Bayern, das Vorliegen identischer Genotyp-Muster. Dies war sowohl bei Verwendung der Restriktionsendonuklease SmaI, als auch bei Einsatz des ApaI-Enzyms (s. Abb. 1, Teil A) der Fall und deutete somit auf eine intra- und interhospitale Verbreitung des gleichen GRE-Stammes.

Die ergänzenden molekularen Untersuchungen (Plasmidmuster und Restriktionsmuster der Plasmide) zeigten, daß die Plasmide in Isolaten mit gleichem Makrorestriktionsmuster eben- falls homogen erschienen (Abb. 1, A und B). Jedoch wiesen die Fragmentmuster der EcoRI-geschnittenen Plasmide deutliche Unterschiede auf (Abb. 1, C, linker Teil). Southern-blot-Hybridisierungen lokalisierten das vanA-Gen in allen Isolaten auf Plasmidfragmenten (Abb. 1 C, rechter Teil).

\section{"GRE-Isolate mit gleichen Makro- restriktionsmustern können sich durch den Besitz unterschiedlicher Resistenzplasmide auszeichnen."}

GRE-Isolate mit gleichen Makrorestriktionsmustern müssen somit nicht zwangsläufig identisch sein, sondern können sich durch den Besitz unterschiedlicher Resistenzplasmide auszeichnen. Die Ergebnisse der Plasmidanalysen weisen ferner darauf hin, daß eine überregionale Ausbreitung von offensichtlich speziell an das Krankenhausmilieu adaptierten E. faeciumStämmen in den einzelnen Krankenhäusern bereits vor Aufnahme der verschiedenen, das vanA-Gencluster tragenden Plasmide erfolgt sein kann.

\section{Literatur}

1. Leclercq R, Derlot E, Duval J, Courvalin P (1988) Plasmid-mediated resistance to vancomycin and teicoplanin in Enterococcus faecium. N Engl J Med 319:157-161

2. Uttley AHC, Collins $\mathrm{CH}$, Naidoo J, George RC (1988) Vancomycin-resistant enterococci. Lancet I:57-58

3. Centers for Disease Control and Prevention (1993) Nosocomial enterococci resistant to vancomycin - United States, 1989-1993. Morbid Mortal Wkl Rep 42:597-599

4. Martone WJ (1998) Spread of vancomycinresistant enterococci: Why did it happen in the United States? Infect Control Hosp Epidemiol 19:539-545

5. Kresken $M$, Hafner $D$ (1988) Prävalenz der Antibiotikaresistenz bei klinisch wichtigen Infektionserregern in Mitteleuropa. Bericht über die Ergebnisse einer multizentrischen Studie der Arbeitsgemeinschaft „Resistenz" in der Paul-Ehrlich-Gesellschaft für Chemotherapie aus dem Jahre 1995. Chemotherapiejournal 5:225-230

6. Wallrauch C, Elsner E, Milatovic D, Cremer J, Braveny I (1997) Antibiotikaresistenz der Enterokokken in Deutschland. Med Klinik 92:464-468
7. Aarestrup FM (1995) Occurrence of glycopeptide resistance among Enterococcus faecium isolates from ecological and conventional poultry farms. Microb Drug Resist 1:255-257

8. Aarestrup FM, Ahrens P, Madsen M, Pallesen LV, Poulsen RL, Westh H (1996) Glycopeptide susceptibility among Danish Enterococcus faecium and Enterococcus faecalis isolates of animal and human origin and PCR identification of genes within the vanA cluster. Antimicrob Agents Chemother 40: 1938-1940

9. Klare I, Heier H, Claus H, Reissbrodt R, Witte W (1995a) vanA-mediated high-level glycopeptide resistance in Enterococcus faecium from animal husbandry. FEMS Microbiol Lett 125: $165-172$

10. Klare I, Heier H, Claus H, Böhme G, Marin S, Seltmann G, Hakenbeck R, Atanassova V, Witte W. (1995) Enterococcus faecium strains with vanA-mediated high-level glycopeptide resistance isolated from animal foodstuffs and faecal samples of humans in the community. Microb Drug Resist 1:265-272

11. Klare I, Badstübner D, Konstabel C, Böhme G, Claus H, Witte W (1999) Decreased incidence of vanA type vancomycin-resistant enterococci isolated from poultry meat and from fecal samples in humans in the community after discontinuation of avoparcin usage in animal husbandry. Microb Drug Resist 5:45-52

12. Witte W, Klare I (1995) Glycopeptide-resistant Enterococcus faecium outside the hospitals: a commentary. Microb Drug Resist 1:259-263

13. Bager F, Aarestrup FM, Madsen $M$, Wegener HC (1999) Glycopeptide resistance in Enterococcus faecium from broilers and pigs following discontinued use of avoparcin. Microb Drug Resist 5:53-56

14. Devriese LA, Pot B, Collins MD (1993) Phenotypic identification of the genus Enterococcus and differentiation of distinct enterococcal species and species groups. J Appl Bacteriol 75:399-408

15. Deutsches Institut für Normung eV (1992) Methoden zur Empfindlichkeitsprüfung von bakteriellen Krankheitserregern (außer Mykobakterien) gegen Chemotherapeutika: Mikrodilution. In:DIN Deutsches Institut für Normung (Hrsg), DIN Taschenbuch 222: Medizinische Mikrobiologie und Immunologie - Normen und weitere Unterlagen. DIN 58940, Teil 8. Beuth, Berlin, S 381-884

16. Klare I, Konstabel C, Bastrop R (1997) Simple and rapid extraction of enterococcal DNA suitable for PCR of vancomycin resistance genes by use of the ion exchanger Chelex 100 Resin. In:Methodische Entwicklungen in der mikrobiologischen Nukleinsäure-Diagnostik, 3./4. Posterworkshop, Dez. 1995/Dez. 1996, Berlin. Hyg Med (Abstract-Band), pp 33-34 
17. Sahm DF, Free L, Handwerger S (1995) Inducible and constitutive expression of vanC1encoded resistance to vancomycin in Enterococcus gallinarum. Antimicrob Agents Chemother 39: 1480-1484

18. Werner G, Klare I, Witte W (1998) Association between quinupristin/dalfopristin resistance in glycopeptide-resistant Enterococcus faecium and the use of additives in animal husbandry. Eur J Clin Microbiol Infect Dis 17: 401-402

19. Werner G, Witte W (1999) Characterization of a new enterococcal gene, sat $G$, encoding a putative acetyltransferase conferring resistance to streptogramin A compounds. Antimicrob Agents Chemother 43: 1813-1814

20. Claus H, Cuny C, Pasemann B, Witte W (1996) A database system for fragment patterns of genomic DNA of Staphylococcus aureus. Zentralbl Bakteriol 287: 105-116

21. Klare I, Heier H, Claus H, Witte W (1993) Environmental strains of Enterococcus faecium with inducible high-level resistance to glycopeptides. FEMS Microbiol Lett 106: 23-30 and Addendum FEMS Microbiol Lett 107:347

22. Tenover FC, Arbeit RD, Goering RV, Mickelsen PA, Murray BE, Persing DH, Swaminathan B (1995) Interpreting chromosomal DNA restriction patterns produced by pulsedfield gel electrophoresis: criteria for strain typing. J Clin Microbiol 33:2233-2239

23. Woodford N, Morrison D, Cookson B, George RC (1993) Comparison of high-level gentamicin-resistant Enterococcus faecium isolates from different countries. Antimicrob Agents Chemother 37:681-684

24. Werner G, Klare I, Witte W (1999) Large conjugative vanA plasmids in vancomycin-resistant Enterococcus faecium. J Clin Microbiol 37:2383-2384

25. Werner G, Klare I, Witte W (1997) Arrangement of the vanA gene cluster in enterococci of different ecological origin. FEMS Microbiol Lett 155:55-61

26. Klare I, Badstübner D, Konstabel C, Witte W (1999) Identification of enterococci and determination of their glycopeptide resistance in German and Austrian clinicalmicrobiological laboratories. Clin Microbiol Infect 5 (in Druck)

27. Hospital Infection Control Practices Advisory Committee, HICPAC (1995) Recommendations for preventing the spread of vancomycin resistance. Morbid Mortal Weekly Rep 4: RR-12

28. Witte W, Heuck D, Klare I, Kniehl E (1996) Stellungnahme $z u_{\text {,"Recommendations for }}$ preventing the spread of vancomycin resistance", erarbeitet durch Hospital Infection Control Practices Advisory Committee (HICPAC), Morbidity and Mortality Weekly Report, September 22, 1995, Vol.4: RR-12. Mikrobiologe 6:134-136
29. Grayson ML, Thauvin-Eliopoulos C, Eliopoulos GM, Yao JDC, deAngelis DV, Walton L, Wolley JL, Moellering RC (1990) Failure of trimethoprim-sulfamethoxazole therapy in experimental enterococcal endocarditis. Antimicrob Agents Chemother 34:1792-1794

30. Norris AH, Reilly JP, Edelstein PH, Brennan PJ, Schuster MG (1995) Chloramphenicol for the treatment of vancomycin-resistant enterococcal infections. Clin Infect Dis 20: 1137-1144

31. Ricaurte JC, Turett GS, Kislak JW (1999) Chloramphenicol treatment for vancomycinresistant Enterococcus faecium bacteremia. In: $9^{\text {th }}$ European Congress of Clinical Microbiology and Infectious Diseases, Berlin, Germany, March 21-24, 1999 (Abstracts). Clin Microbiol Infect 5 [Suppl 3] p 74 (Abstract P146)

32. Lukashok SA, Casadevall A (1995) Persistent vancomycin-resistant Enterococcus faecium bacteremia. In: Abstracts of the $35^{\text {th }}$ Interscience Conference on Antimicrobial Agents and Chemotherapy, San Francisco LA, 1995. American Society for Microbiology, Washington DC, p 270 (Abstract J74)

33. Bryson HM, Spencer CM (1996) Quinupristindalfopristin. Drugs 52:406-415

34. Hill RLR, Smith CT, Seyed-Akhavani M, Casewell MW (1997) Bactericidal and inhibitory activity of quinupristin/dalfopristin against vancomycin- and gentamicin-resistant Enterococcus faecium. J Antimicrob Chemother 39 [Suppl A]:23-28

35. Low DE (1995) Quinupristin/dalfopristin: spectrum of activity, pharmacokinetics, and initial clinical experience. Microb Drug Resist 1: 223-234

36. Thal LA, Zervos MJ (1999) Occurrence and epidemiology of resistance to virginiamycin and streptogramins.J Antimicrob Chemother 43: 171-176

37. Hummel R, Tschäpe H, Witte W (1986) Spread of plasmid-mediated nourseothricin resistance due to antibiotic use in animal husbandry. J Basic Microbiol 26:461-466

38. Tschäpe $H$ (1994) The spread of plasmids as a function of bacterial adaptibility. FEMS Microbiol Lett 15:23-32

39. Reinert RR, Conrads G, Schlaeger JJ, Werner G, Witte W, Lütticken R, Klare I (1999) Survey of antibiotic resistance among enterococci in North-Rhine Westphalia, Germany.J Clin Microbiol 37: 1638-1641

40. Klare I, Witte W, Reinhardt A, Just HM, Eßinger U, Höffler D (1996) Ausbrüche von Infektionen mit vanA-positiven high-level glycopeptidresistenten Enterococcus faecium (VRE) in Deutschland. In: 48. Jahrestagung der Deutschen Gesellschaft für Hygiene und Mikrobiologie eV, Bonn, 8.-11.0ktober 1996 (Abstract-Band): p 117 (Abstract P154)

41. Klare I, Witte W (1997) Glycopeptidresistente Enterokokken: zur Situation in Deutschland. Hyg Mikrobiol 2:31-38

42. Klare I, Witte W (1997) Glycopeptidresistente Enterokokken: Auftreten, Verbreitung, Resistenzübertragung, Bedeutung. Wien Klin Wochenschr 109:293-300
In den vergangenen Wochen erreichten uns die unten aufgeführten Neuankündigungen. Ausgewählte Titel werden in nächster Zeit besprochen.

\section{F. Hofmann}

Infektiologie

Landsberg: ecomed, 1999. Ca. 2.500 S., Loseblattwerk in 3 Leinenordnern, (ISBN 3-609-71540-5), DM 248,-

\section{H. Lode, W. Siegenthaler}

Neue Erkenntnisse in der Infektiologie Stuttgart, New York: Thieme, 1999. 226 S., 35 Abb., 19 Tab., (ISBN 3-13-105181-7/694), kart., DM 99,-

\section{H.-J.Tietz, W. Sterry}

Antimykotika von A-Z

Berlin, Wien, Melbourne: Blackwell, 1999.112 S., (ISBN 3-89412-367-2), brosch., DM 38,-

\section{J.Woolliscroft}

Diagnose- und Therapielexikon für den Hausarzt

Berlin, Heidelberg, New York: Springer, 1999. 500 S., 119 Abb., (ISBN 3-540-64474-1), brosch., DM 79,-

\section{E. Hoffmann, G. Steinbeck \\ Interventionelle kardiale}

Elektrophysiologie

Berlin, Heidelberg, New York: Springer, 1999. 328 S., 120 Abb., (ISBN 3-540-65684-7), geb., DM 198,- 